\title{
Los focus groups como herramienta en métodos cualitativos: experiencias contrastadas en docencia e investigación
}

\author{
Blanca de-Miguel-Molina ${ }^{a}$, María de-Miguel-Molina ${ }^{\mathrm{b}}$, Virginia Santamarina- \\ Campos $^{\mathrm{c}}$, y Daniel Catalá-Pérez ${ }^{\mathrm{d}}$ \\ ${ }^{a}$ Departamento de Organización de Empresas, Universitat Politècnica de València, \\ bdemigu@omp.upv.es, 'bepartamento de Organización de Empresas, Universitat Politècnica de \\ València, mademi@omp.upv.es, ${ }^{\mathrm{c}}$ Departamento de Conservación y Restauración de Bienes \\ Culturales, Universitat Politècnica de València, virsanca@crbc.upv.es, ${ }^{\mathrm{d}}$ Departamento de \\ Organización de Empresas, Universitat Politècnica de València, dacape@ade.upv.es
}

\begin{abstract}
Qualitative methods let us to obtain more precise information about the users's needs of a product or service. In order to gather this information, we can apply different tools such as focus groups. The creation of these groups is quite simple to perform in the classroom and, while students learn this tool, they can share information relevant to any aspect of their professional career. In this case, we have transferred the experience of this tool within a European Project (H2020) to an activity to be carried out in the classroom within the subject Research Methodologies. Finally, a content analysis was carried out with the transcriptions of these focus groups, and the feedback of the results was given to the student-participants and professors of the master's degree.
\end{abstract}

Keywords: research methodologies, qualitative methods, focus groups, content analysis.

\begin{abstract}
Resumen
Los métodos cualitativos permiten obtener una información más completa de los usuarios de un producto o servicio, para lo que se sirven de distintas herramientas como los focus groups. La creación de estos grupos es relativamente sencilla de realizar en el aula y, a la vez que los alumnos aprenden esta herramienta, pueden compartir información relevante para cualquier aspecto de su carrera profesional. En este caso, nosotros hemos trasladado la experiencia de esta herramienta dentro de un proyecto europeo (H2020), a una actividad a realizar en el aula dentro de la asignatura Metodologías de Investigación (Research Methodologies). Posteriormente, se realizó un análisis de contenido con la información transcrita y se dio a los alumnos-participantes y profesores del máster el feedback de los resultados.
\end{abstract}

Palabras clave: metodologías de investigación, métodos cualitativos, focus groups, análisis de contenido. 


\section{Introducción}

Conocer las necesidades de los usuarios a la hora de crear nuevos productos o servicios, o mejorarlos, no es una tarea sencilla. En ocasiones, podemos encontrar fuentes de información secundaria que nos faciliten dicha información pero, en otras, necesitamos nuestras propias fuentes de información para adaptar ese producto/servicio de la mejor manera posible.

En la asignatura Research Methodologies, del Máster Universitario en Gestión de Empresas, Productos y Servicios, les explicamos a los alumnos distintas metodologías de investigación cualitativa y las herramientas de las que se pueden servir para emplearlas. Además, sus aplicaciones en la empresa pueden ser variadas, no sólo para conocer el punto de vista de los clientes, sino incluso para conocer necesidades internas de sus empleados.

Entre esos métodos cualitativos encontramos el análisis de contenido, que Zhang y Wildemuth (2017) definen como "ir más allá de contar palabras o extraer contenido objetivo de los textos, para examinar significados, temas o patrones que pueden ser manifiestos o estar latentes en un texto. Permite a los investigadores entender una realidad social de una manera subjetiva pero científica”.

Por otro lado, en nuestra propia investigación en un proyecto europeo del Horizonte 2020, para obtener información primaria y realizar un análisis de su contenido, hemos utilizado la herramienta de focus groups. Ello nos ha permitido conocer las necesidades de una industria concreta y así poder desarrollar un producto acorde a éstas.

El focus group lo definen Berg y Lune (2012) como un grupo pequeño de discusión sobre un tema concreto, coordinado por un moderador, siendo ese tema relevante tanto para el investigador como para el grupo.

De este modo, nos planteamos que podíamos no solo explicar a los alumnos la metodología (análisis de contenido) y la herramienta (focus groups), sino que además fuesen ellos los partícipes de la actividad. Y para esto, buscamos unos objetivos en los que pudiesen participar de manera activa.

\section{Objetivos}

El objetivo de la actividad como tal, fue analizar las necesidades específicas de los alumnos del Máster para mejorar el programa, teniendo en cuenta que la mayoría de nuestros alumnos son internacionales.

Con ello, además, lográbamos otros dos objetivos:

- Que los alumnos conociesen la herramienta de los focus groups y cómo se analiza después esa información por medio del análisis de contenido.

- Que los alumnos intercambiasen sus experiencias y se creara un "debate” sobre los temas tratados. 


\section{Desarrollo de la innovación}

La actividad se llevó a cabo en diciembre de 2017 y contó con dos facilitadores, uno por grupo. Se realizó en un aula de la Facultad de Administración y Dirección de Empresas de la UPV, con una duración aproximada de una hora.

Los participantes fueron una parte de los alumnos del máster (la mitad más o menos), tanto hombres como mujeres de distintas nacionalidades. En total, 10 alumnos repartidos en dos grupos de 5. En cada grupo, además, uno de los alumnos hizo de soporte al facilitador para ir transcribiendo los comentarios de los participantes y así no ser necesario grabar la sesión. Para formar los dos grupos, el criterio seguido fue el de la lengua nativa, siendo un grupo de castellanoparlantes y el otro de distintos idiomas de lengua materna (ruso, holandés, alemán, árabe, entre otros). De todos modos, el idioma en el que se realizaron los focus groups fue el inglés al ser la lengua en la que se imparte la asignatura y conocida por todos los alumnos.

Sin embargo, en sesiones más largas o donde no se tomen notas por otra persona, es conveniente la grabación del audio (y si es posible de la imagen) para después transcribir todos los comentarios. En ese caso, es preciso entregar a los participantes un acuerdo para firmar su consentimiento sobre la grabación, siguiendo las pautas de la normativa sobre protección de datos (que, a partir del 25 de mayo de 2018, se basará en el Reglamento Europeo de Protección de Datos de 2016) (Unión Europea, 2016). En cualquier caso, la información a tratar era confidencial, sin citar en ningún momento a los intervinientes.

La dinámica se preparó con la misma base de los focus groups realizados en el proyecto H2020 citado (AiRT Project, 2017) (Imagen 1), preparando previamente un guión para ir realizando las preguntas a los participantes sobre los temas en los que se quería profundizar y así conocer las necesidades de los estudiantes de este máster.

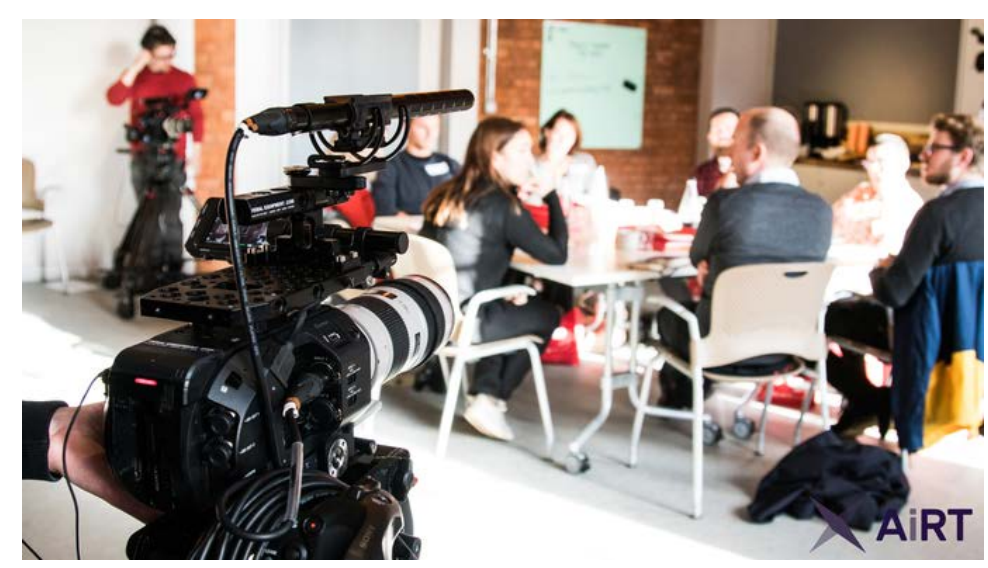

Imagen 1. Focus groups desarrollados en UK durante el Proyecto AiRT.

Fuente: repositorio del proyecto. 
Para preparar el cuestionario, se seleccionaron como temas básicos:

- La motivación de los estudiantes para escoger este máster.

- $\quad$ La experiencia que están viviendo durante su realización.

- $\quad$ Sus planes de futuro.

- Cómo les puede ayudar el máster a desarrollar su carrera profesional.

Para desplegar estos puntos, finalmente se elaboraron 12 preguntas. Estas fueron consensuadas entre algunos profesores, antiguos alumnos y la dirección del máster.

Finalizado el focus groups, se repasaron las notas entre el facilitador, el ayudante y los participantes, para comprobar que toda la información relevante estaba incluida. Posteriormente, con la información de ambos grupos, se realizó una análisis de contenido para codificar la información.

Para terminar, se elaboraron las conclusiones y se hicieron extensibles a todos los alumnos participantes y profesores del máster. Todos los datos fueron tratados de forma anónima.

\section{Resultados}

Los alumnos participaron de manera muy activa durante toda la dinámica, exponiendo sus impresiones y experiencias, lo que fue muy enriquecedor para los alumnos y profesores.

Sin lugar a dudas, el atractivo de Valencia como ciudad y el que la mayoría del programa sea en inglés, son factores atrayentes para los aumnos.

Entre los problemas encontrados, muchos están relacionados con la burocracia, especialmente para los estudiantes internacionales.

Lo que más se valora del máster es la experiencia en su conjunto, no solo por lo que aporta un título de alto nivel, sino también porque les aporta valor en su carrera profesional, destacando el entorno multidiciplinar en el que se desenvuelven.

Los resultados exactos de estos focus groups son información valiosa para nuestros competidores, por lo que no podemos detallar los resultados específicos de cada pregunta.

El guión que siguió el facilitador fue proporcionado posteriormente a los alumnos, de manera que conociesen cómo se había planificado la dinámica. Asimismo, como ya se había impartido otra sesión sobre análisis de contenido, al explicar el estudio realizado los conocimientos quedaron más asentados.

Las conclusiones se difundieron entre los alumnos-participantes y se les dio la posibilidad de aclarar o puntualizar algo si lo creían necesario.

Además, estas conclusiones se compararon con las del estudio realizado para QS por Karzunina et al. (2016), coincidiendo en varias de ellas, por ejemplo:

- $\quad$ El impacto que puedan tener sus estudios en la empleabilidad.

- Los problemas que se encuentran para acceder a determinada información proporcionada por las universidades.

(cc) EY-NC-ND 2018, Universitat Politècnica de València

Congreso IN-RED (2018) 
- $\quad$ La importancia de la posición que tenga a universidad a nivel internacional.

\section{Conclusiones}

Tras concluir esta actividad, comprobamos que todos los objetivos que nos habíamos propuesto se habían cubierto. En primer lugar, el objetivo de la actividad específica quedó completado con el análisis de las necesidades concretas de los alumnos del Máster.

El objetivo académico y principal, que era que los alumnos conociesen la herramienta de los focus groups y cómo se analiza después esa información por medio del análisis de contenido, se completó de una manera activa, siendo un tema interesante tanto para los alumnos como para los profesores de la asignatura que actuaron de facilitadores. Así mismo, el feedback continuo durante la actividad permitió que los alumnos conociesen la dinámica en caso de que tengan que replicarla en el futuro para sus investigaciones.

Por último, y aunque fuese un objetivo paralelo, los alumnos agradecieron que esta actividad les permitiese intercambiar sus experiencias y se creara un "debate” sobre los temas tratados. Al estar presentes los profesores como facilitadores, se sintieron escuchados y que su aportación puede contribuir a mejorar el programa.

De este modo, podemos concluir que existen herramientas de investigación, como los focus groups, que pueden aplicarse tanto en la docencia como en la investigación y, de esta manera, conocer una aplicación real que puede servir en las metodologías cualitativas. En definitiva, la actividad ha contribuido al objetivo final de la asignatura que es que los alumnos conozcan las bases de las metodologías de investigación en Ciencias Sociales.

\section{Agradecimientos}

Este trabajo se realizó en el marco del Proyecto de Innovación y Mejora Educativa (PIME), “La resolución de problemas a través del diseño creativo y la visualización colaborativa” de la UPV, coordinado por la profesora V. Santamarina. Asimismo, la metodología está siendo aplicada en el proyecto de investigación "Technology Transfer Of Remotely Piloted Aircraft Systems (Rpas) For The Creative Industry (AiRT)”, de la Comisión Europea (H2020).

\section{Referencias}

AiRT PROJECT. Technology Transfer Of Remotely Piloted Aircraft Systems (Rpas) For The Creative Industry. http://www.airt.eu [Consulta: 1 de febrero de 2018]

BERG, B.L. y LUNE, H. (2012). Qualitative Research Methods for the Social Sciences. New Jersey: Pearson.

Unión Europea. Reglamento del Parlamento Europeo y del Consejo, relativo a la protección de las personas físicas en lo que respecta al tratamiento de datos personales y a la libre circulación de estos datos y por el que se deroga la Directiva 95/46/CE (Reglamento general de protección de datos). Diario Oficial de la Unión Europea, de 27 de abril de 2016, núm. 679, p. L 119/1-L 119/88. 
Los focus groups como herramienta en métodos cualitativos: experiencias contrastadas en docencia e investigación

KARZUNINA, D. ; BRIDGESTOCK, L. y PHILIPPOU, G. (2016). What matters to international students. London: QS Intelligence Unit.

ZHANG, Y. y WILDEMUTH, B.M. (2016). “Qualitative analysis of content“ en Wildemuth, B.M. Applications of social research methods to questions in information and library science. Santa Bárbara, California : Unlimited Libraries.

(cc) EY-NC-ND 2018, Universitat Politècnica de València 